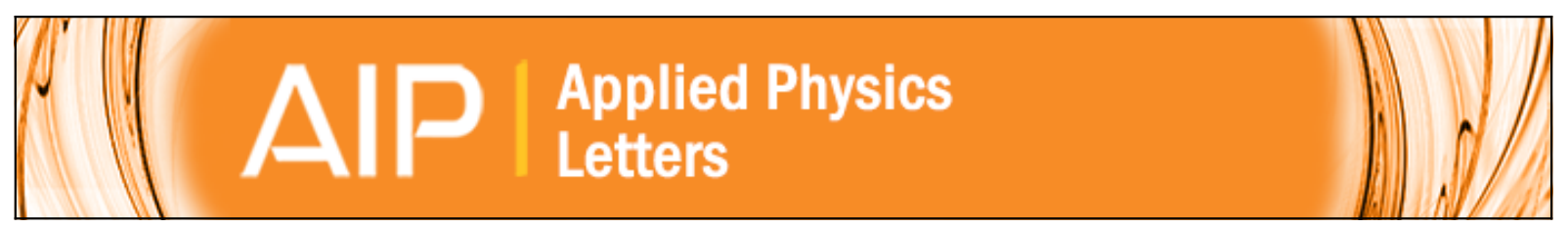

\title{
Terahertz focusing of multiple wavelengths by graphene metasurfaces
}

Liming Liu, Yair Zarate, Haroldo T. Hattori, Dragomir N. Neshev, llya V. Shadrivov, and David A. Powell

Citation: Applied Physics Letters 108, 031106 (2016); doi: 10.1063/1.4940231

View online: http://dx.doi.org/10.1063/1.4940231

View Table of Contents: http://scitation.aip.org/content/aip/journal/apl/108/3?ver=pdfcov

Published by the AIP Publishing

\section{Articles you may be interested in}

Magneto-optic transmittance modulation observed in a hybrid graphene-split ring resonator terahertz metasurface

Appl. Phys. Lett. 107, 121104 (2015); 10.1063/1.4931704

Dual-gated tunable absorption in graphene-based hyperbolic metamaterial

AIP Advances 5, 067106 (2015); 10.1063/1.4922170

Hybrid metasurface for ultra-broadband terahertz modulation

Appl. Phys. Lett. 105, 181108 (2014); 10.1063/1.4901050

Tunable terahertz left-handed metamaterial based on multi-layer graphene-dielectric composite Appl. Phys. Lett. 104, 051902 (2014); 10.1063/1.4863929

Dynamically tunable broadband mid-infrared cross polarization converter based on graphene metamaterial Appl. Phys. Lett. 103, 223102 (2013); 10.1063/1.4833757

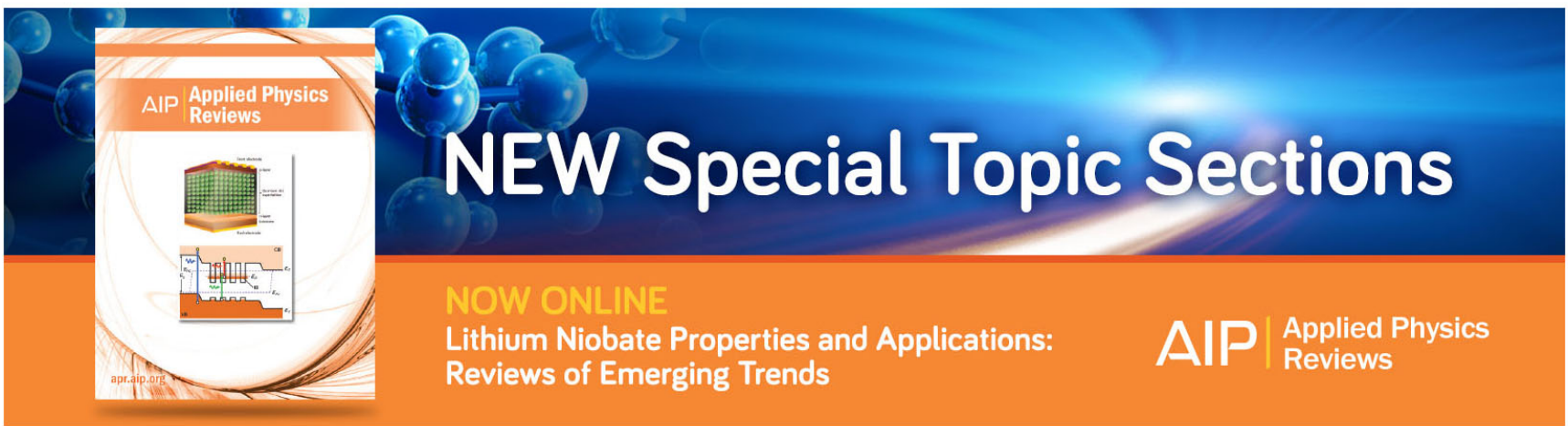




\title{
Terahertz focusing of multiple wavelengths by graphene metasurfaces
}

\author{
Liming Liu, ${ }^{1,2, a)}$ Yair Zarate, ${ }^{1}$ Haroldo T. Hattori, ${ }^{2}$ Dragomir N. Neshev, ${ }^{1,3}$ Ilya V. Shadrivov, ${ }^{1}$ \\ and David A. Powell ${ }^{1,3}$ \\ ${ }^{1}$ Nonlinear Physics Centre, Research School of Physics and Engineering, Australian National University, \\ Canberra, ACT 2601, Australia \\ ${ }^{2}$ School of Engineering and Information Technology, University of New South Wales, Canberra, ACT 2612, \\ Australia \\ ${ }^{3}$ Centre for Ultrahigh Bandwidth Devices for Optical Systems (CUDOS), Australian National University, \\ Canberra, ACT 2601, Australia
}

(Received 15 October 2015; accepted 7 January 2016; published online 20 January 2016)

\begin{abstract}
Metasurfaces can achieve nearly arbitrary wavefront control based on manipulation of the wave phase profile. We propose a metasurface based on double graphene cut-wire resonators which can cover the complete $2 \pi$ phase region with high reflection efficiency. This full phase coverage is essential for efficient wavefront manipulation, without reflecting energy into unwanted channels. A mirror capable of focusing multiple wavelengths is demonstrated numerically based on the proposed structure. The mirror can effectively focus terahertz (THz) waves from 1.2 to $1.9 \mathrm{THz}$ to the same focal point by changing the Fermi level of each graphene resonator separately. The presented metasurface could provide a powerful platform for controlling $\mathrm{THz}$ waves, including focusing, beam steering, beam shaping, and holography. (C 2016 AIP Publishing LLC.

[http://dx.doi.org/10.1063/1.4940231]
\end{abstract}

Metasurfaces, a type of two-dimensional metamaterials, have proven to be a simple but effective way to control the amplitude, phase, and polarization of electromagnetic waves. ${ }^{1}$ Interesting functionalities have been proposed and demonstrated, including anomalous reflection or refraction, ${ }^{2}$ flat lens, ${ }^{3}$ and holography ${ }^{4,5}$ based on metasurfaces with full phase modulation.

Large phase modulation is usually associated with resonance shift and implemented by tunable resonant metamaterials. Various approaches have been explored to accomplish tunable metamaterials in various frequency ranges. In particular, great efforts have been placed in the terahertz $(\mathrm{THz})$ range, where there is a high demand for wave manipulating devices. For $\mathrm{THz}$ metamaterials, tunability can be achieved by using materials like semiconductors ${ }^{6}$ or liquid crystals, ${ }^{7}$ the properties of which can be changed by applying voltage or by changing the temperature of the device. Alternative ways to get larger resonance tunability include changing the geometry directly or manipulating the coupling between adjacent metamaterial layers in multi-layer metamaterials. ${ }^{8,9}$

Compared with uniformly tunable metamaterials, it is more challenging to implement a gradient metasurface with dynamic phase tunability. For such metasurfaces, it is necessary to control the unit cells individually to produce the required phase shift. However, no such metasurfaces with dynamical gradient phase control have been demonstrated to date due to the complexity of fabrication.

Graphene could provide an alternative solution for metasurfaces with dynamic phase control. Unlike the quasi dielectric behavior of graphene at optical frequencies, graphene shows strong metallic properties determined by intra-band transitions at $\mathrm{THz}$ frequencies. ${ }^{10}$ The conductivity of graphene

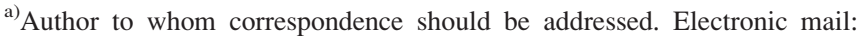
liming.liu@student.adfa.edu.au
}

can be determined from its Fermi level through the Kubo formula. ${ }^{11}$ The Fermi level $E_{f}$ can be tuned by applying gating voltages $V_{g}$ on graphene to change its carrier density, which is determined by $\left|E_{f}\right|=\hbar V_{f} \pi^{1 / 2}\left(n_{0}^{2}+\alpha^{2}\left|V_{C N P}-V_{g}\right|^{2}\right)^{1 / 4} \cdot{ }^{12}$ The Fermi velocity is $V_{f} \approx 1 \times 10^{6} \mathrm{~m} / \mathrm{s}, n_{0}$ is the residual carrier concentration which is varied across different graphene samples, $\alpha$ is the gate capacitance determined by the specific electrode configuration, and $V_{C N P}$ is the charge neutral point where numbers of electrons and holes are equal. Fitting parameters from experimental results are required ${ }^{12}$ to calculate the gating voltage $V_{g}$ which has been reported from several volts ${ }^{13,14}$ to hundreds of volts ${ }^{12}$ experimentally. In order to avoid overwhelming readers but still address the underlying physics, Fermi levels instead of voltages are used in the text, which is the same as in other studies. ${ }^{15-17}$

This tunable conductivity of graphene has inspired researchers to explore the possibility of replacing metals by graphene in tunable metamaterials. ${ }^{13,15,18,19}$ For graphene based metasurfaces operating in reflection, previous works have focused on the TM excited plasmon resonances in infinitely long graphene ribbons. ${ }^{16,17}$ For example, beam steering, focusing, and non-diffracting Airy beams have been demonstrated based on the graphene ribbons. ${ }^{16}$ However, a complete $2 \pi$ phase modulation cannot be fully covered only by adjusting the width or Fermi level of those graphene ribbons. ${ }^{16}$ Furthermore, the configuration of a one-dimensional infinitely long graphene ribbon could not be directly used in two dimensional metasurfaces.

In this letter, we propose a reflective metasurface with double graphene cut-wire resonators (DGCR) as the building block. Each graphene cut-wire resonator has a fundamental electric dipole mode, which is known to provide stronger graphene-light interactions than magnetic or other higherorder modes. ${ }^{20}$ By arranging two different graphene cut-wire resonators in one unit cell, two resonances can be excited. 
As the two resonant frequencies are determined by the Fermi level of the graphene resonators, two independently controllable Fermi levels are in principle available for tuning the structure, as compared with the conventional graphene ribbon which only has one controllable parameter after fabrication, i.e., either ribbon width or Fermi level.

The DGCR unit cell consists of an optically thick gold ground plane and two atomically thin graphene cut-wires separated by a dielectric layer, and two electrodes for gating each graphene resonator as shown in Fig. 1(a). The graphene cut-wires have the same width $a=15 \mu \mathrm{m}$ but different lengths $b_{1}=24 \mu \mathrm{m}$ and $b_{2}=19 \mu \mathrm{m}$ to produce two resonances. Developing electrodes with a large electrical conductivity and high $\mathrm{THz}$ transparency to gate graphene is the most critical step to achieve tunability experimentally. By using deep-subwavelength metallic electrode arrays ${ }^{12}$ and ion-gel top gates, ${ }^{13}$ graphene based $\mathrm{THz}$ devices can work effectively without significant perturbation of their electromagnetic response. A better candidate for gating graphene is Indium-Tin-Oxide (ITO) Nanowhiskers (NWhs) which have large electrical conductivity and high $\mathrm{THz}$ transparency of $82 \%$ up to $15 \mathrm{THz}^{21,22}$ Electron beam lithography can be used to pattern the ITO NWhs electrodes and it is even possible by using an inherent micro-masking mechanism during the RIE process ${ }^{23}$ due to the presence of indium.

Numerical simulations are performed by using commercial electromagnetic solver CST Microwave Studio with a frequency domain analysis. Unit cell boundary is applied in both the $\mathrm{X}$ and $\mathrm{Y}$ directions while the $\mathrm{Z}$ direction has an open boundary. We assume that the dielectric is a $10 \mu \mathrm{m}$ thick benzocyclobutene (BCB), with permittivity $\epsilon=2.67$ and loss tangent of $0.012 .^{24}$ We have used a surface impedance approach to model graphene of vanishing thickness in CST, which uses the complete Kubo formula ${ }^{11}$ for conductivity. To validate this approach, we have run simulations and reproduced the results for the graphene ribbon. ${ }^{25}$ The temperature of graphene is $300 \mathrm{~K}$ and phenomenological scattering rate is assumed to be $0.11 \mathrm{meV} .^{11}$
For an incident $\mathrm{THz}$ wave with electric field polarized in the $\mathrm{Y}$ direction, two plasmon resonances are excited at 1.27 THz and 1.48 THz as shown in the red line of Fig. 1(b). To study the effects of gating electrodes on the THz performance of the device, complex permittivity of ITO NWhs is taken from experimental results. ${ }^{22}$ It is shown in the blue line of Fig. 1(b) that adding the ITO NWhs electrode has negligible effects on the $\mathrm{THz}$ performance of the device. Further simulations are conducted for unit cell with only long (dashed line) or short (dotted dashed line) resonator with electrodes, and the results indicate that no clear coupling is observed between the two resonators in the DGCR. Almost $2 \pi$ phase variation is produced in each resonance due to the additional metallic ground plane, ${ }^{16,26}$ as shown in the phase profile of Fig. 1(b).

As the plasmon resonance $\omega_{p}$ of graphene resonators is mainly determined by the resonator length $b$ and Fermi level $E_{f}$, we have examined the quantitative relation between $\omega_{p}$ and the two parameters. Data points in Fig. 1(c) show the excitation of $\omega_{p}$ when increasing Fermi levels from 0.1 to $0.9 \mathrm{eV}$ for different resonator length of $b=15 \mu \mathrm{m}$, $b=19 \mu \mathrm{m}$, and $b=24 \mu \mathrm{m}$, respectively. A square root scaling behavior $\omega_{p} \propto\left|E_{f}\right|^{1 / 2}$ can be used to rather accurately fit those data points, which is an indication of massless Dirac electrons. ${ }^{13}$ However, an inverse square root power-law scaling behavior $\omega_{p} \propto b^{-1 / 2}$ is governing the relation between $\omega_{p}$ and resonator length $b$ as shown in Fig. 1(d), which reveals the signature of $2 \mathrm{D}$ electron systems. ${ }^{27}$

For efficient wavefront manipulation, a continuous $2 \pi$ phase modulation is crucial. Figs. 2(a) and 2(b) depict the reflection phase at $1.45 \mathrm{THz}$ and $1.7 \mathrm{THz}$, when varying the Fermi level of the two cut-wire resonators from 0.3 to $0.9 \mathrm{eV}$. From the phase color map, it is clear that, by fixing the Fermi level of the short resonator, e.g., at $0.5 \mathrm{eV}$ (white dot line), almost $2 \pi$ phase is achieved by varying the long resonator from 0.3 to $0.9 \mathrm{eV}$ at $1.45 \mathrm{THz}$. It is worth noting that the minimum reflection amplitude reaches $48 \%$ corresponding to the selected phase line. However, at $1.7 \mathrm{THz}$ the (a)
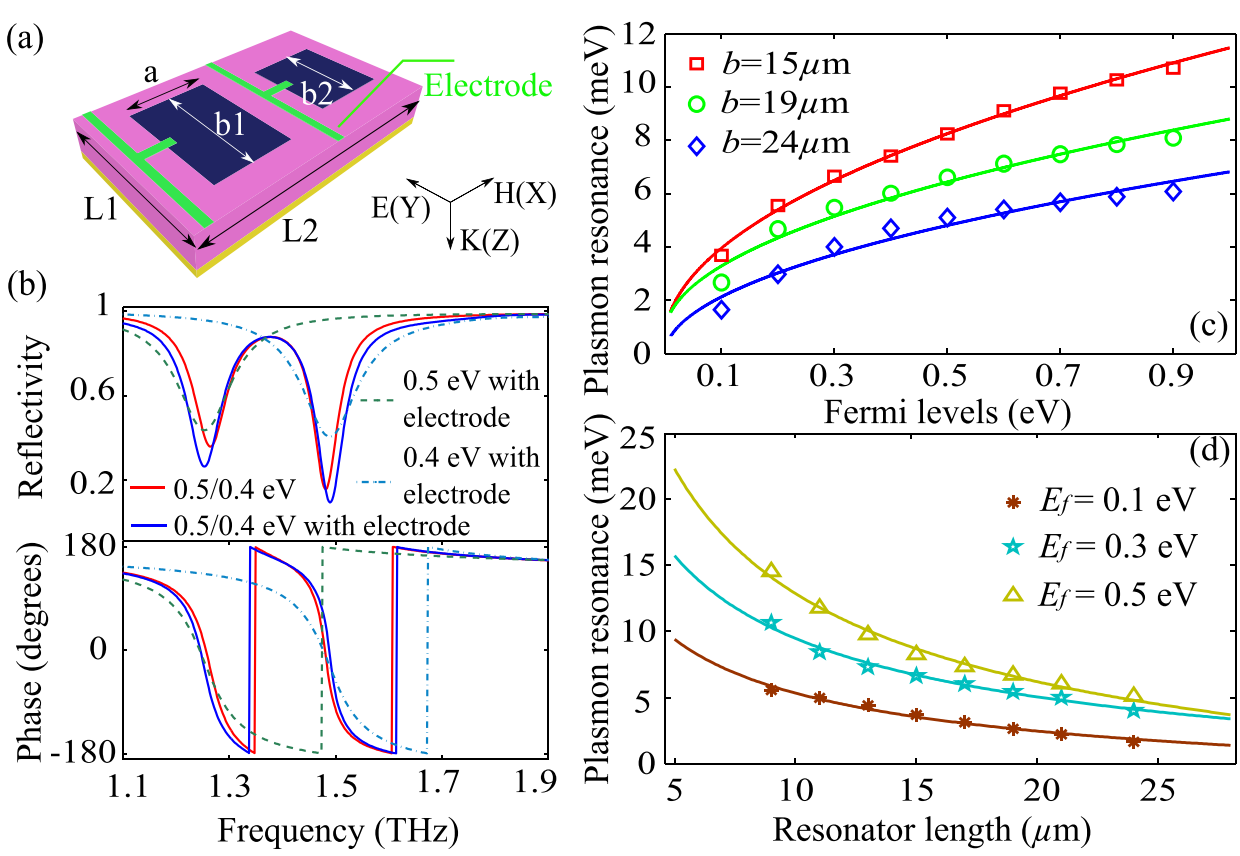

FIG. 1. (a) Schematics of the proposed DGCR with dimensions of $L 1=28$ $\mu \mathrm{m}, L 2=40 \mu \mathrm{m}$ and ITO NWhs electrode of width $1 \mu \mathrm{m}$ and thickness $100 \mathrm{~nm}$. (b) Reflectivity and phase profile for the Fermi levels of $0.5 / 0.4 \mathrm{eV}$ without (red line) and with (blue line) ITO NWhs electrodes. Unit cells with only long (dashed line) or short resonator (dotted dashed line) indicate that there is no coupling between the two resonators in the DGCR. Plasmon resonances (in $\mathrm{eV}$ unit) versus the (c) Fermi levels and (d) resonator lengths. The solid lines are square root fits to the numerical points. 

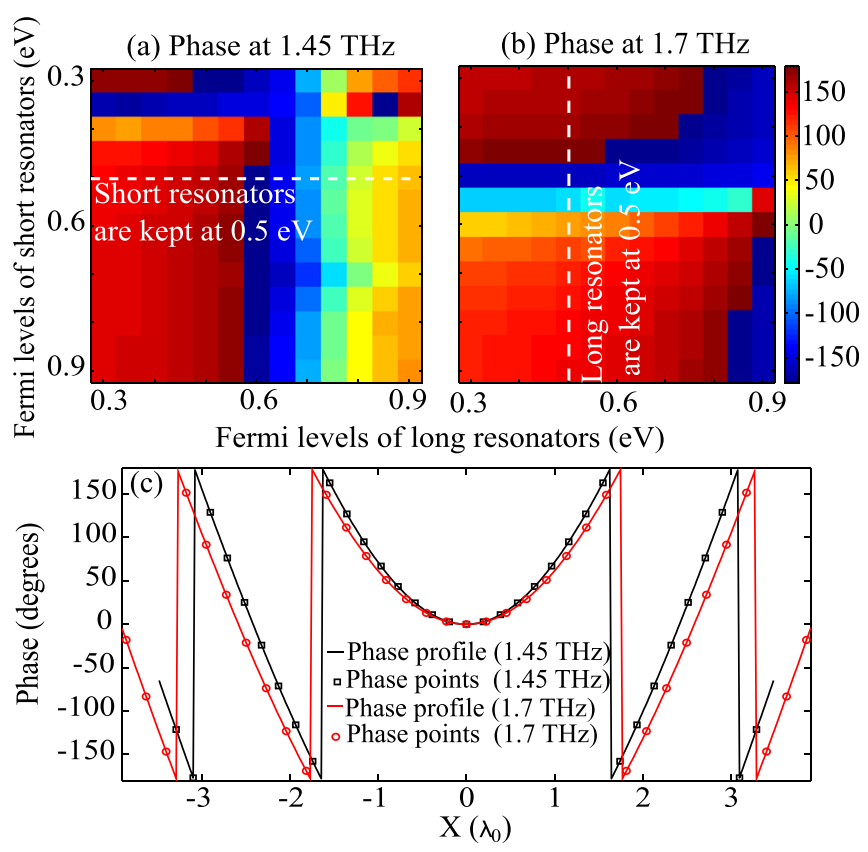

FIG. 2. Phase color map for (a) $1.45 \mathrm{THz}$ and (b) $1.7 \mathrm{THz}$ when varying Fermi levels between 0.3 and $0.9 \mathrm{eV}$ for both resonators. (c) The desired phase profile for $1.45 \mathrm{THz}$ (black line) and $1.7 \mathrm{THz}$ (red line) with the 35 corresponding phase points used to approximate the phase profile.

$2 \pi$ phase modulation has to be implemented by fixing the long cut-wire resonator, e.g., at $0.5 \mathrm{eV}$ and varying the short resonator, which shows an opposite phase modulation profile of that at $1.45 \mathrm{THz}$. This is an important achievement as it provides $2 \pi$ phase coverage in a large frequency range by only changing the Fermi levels of graphene. To obtain similar results, previous designs based on graphene ribbons have to vary both Fermi level and ribbon width, ${ }^{17}$ hence after fabrication these structures are not dynamically tunable.

Using the continuous $2 \pi$ phase modulation achieved above, we calculate a focusing mirror as a proof of concept. For a focusing mirror, $\mathrm{THz}$ waves reflected from each DGCR must have the same phase at the focal point interface constructively. This requires each DGCR to produce a different phase response to compensate the propagation phase between its own location and the focal point. For a designed focal length $f$ at wavelength $\lambda_{0}$, the phase distribution $\psi(X)$ along the interface is defined as

$$
\psi(X)=\frac{2 \pi}{\lambda_{0}}\left(\sqrt{X^{2}+f^{2}}-f\right),
$$

in which $\lambda_{0}(207 \mu \mathrm{m}$ for $1.45 \mathrm{THz})$ is the working wavelength and $X(\mu \mathrm{m})$ is the position of the DGCR cell.

The desired phase profile calculated from Eq. (1) for a focusing mirror with $500 \mu \mathrm{m}$ focal length at $1.45 \mathrm{THz}$ is depicted as a black line in Fig. 2(c). A total of 35 phase points shown as black squares are used to approximate the phase profile. Figs. 3(a)-3(c) show electric field recorded at $1.2,1.45$, and $1.7 \mathrm{THz}$ for a focusing mirror designed working at $1.45 \mathrm{THz}$. In contrast to the strong focusing effect at $1.45 \mathrm{THz}$ shown in Fig. 3(b), no clear focusing effect is observed at 1.2 and $1.7 \mathrm{THz}$ revealed in Figs. 3(a) and 3(c), which is due to the resonant structures used for building metasurfaces. However, it is always desirable for a lens

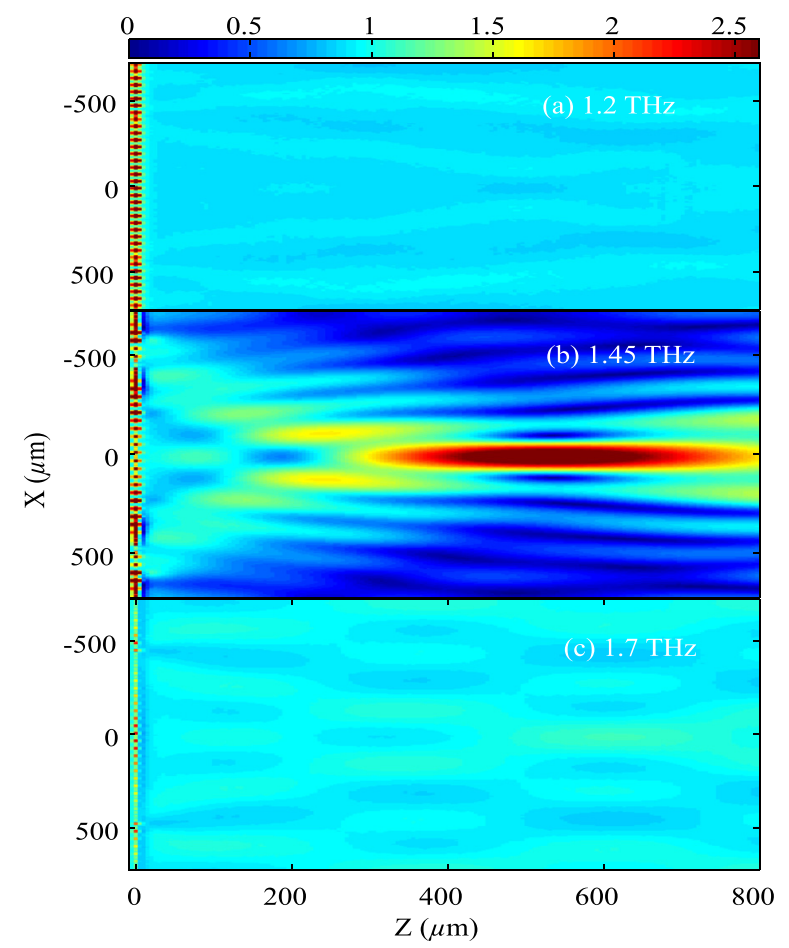

FIG. 3. Electric field amplitude recorded at (a) $1.2 \mathrm{THz}$, (b) $1.45 \mathrm{THz}$, and (c) $1.7 \mathrm{THz}$ for focusing mirror with desired phase profile for $1.45 \mathrm{THz}$ with the field of the incident waves subtracted.

being able to focus multiple wavelengths in the same spot. To achieve this, the entire resonating metasurface needs to be dynamically tuned.

Referring to Eq. (1) when working at a different frequency, the corresponding phase profile needs to be changed accordingly. The red line in Fig. 2(c) gives the required phase profile for $1.7 \mathrm{THz}$ to have the same focal length of $500 \mu \mathrm{m}$. The red circles are 35 phase points used to approximate the phase profile at $1.7 \mathrm{THz}$. Figs. 4(a)-4(d) show the E-field plot for the frequencies of 1.2, 1.45, 1.7, and 1.9 $\mathrm{THz}$, respectively, after having the desired phase profiles. With dynamical phase modulation, the mirror effectively focuses different $\mathrm{THz}$ waves into almost the same focal point. To confirm the focusing effect, the E field along the symmetry axis of $Z$ direction is extracted $(X=0 \mu \mathrm{m})$ and it is shown in Fig. 5(a). The electric field reaches its maximum at $\mathrm{Z}=525 \mu \mathrm{m}, 525 \mu \mathrm{m}, 530 \mu \mathrm{m}$, and $510 \mu \mathrm{m}$ for $1.2,1.45$, 1.7, and $1.9 \mathrm{THz}$, respectively. This small discrepancy (around 4\%) compared with the designed focal length $\mathrm{Z}=500 \mu \mathrm{m}$ mainly comes from numerical approximation and finite size of the unit cell. The electric field distribution of the metasurface parallel to the mirror interface (X direction) is also shown in Fig. 5(b) when $\mathrm{Z}$ is fixed to its own focal points for each frequency. The spot size is found by fitting the cross-sectional E-field intensity of metasurface to its $e^{-1}$ full-width. ${ }^{28}$ The calculated spot sizes are $0.77 \lambda_{0}$, $0.71 \lambda_{0}, 0.71 \lambda_{0}$, and $0.79 \lambda_{0}$ for $1.2,1.45,1.7$, and $1.9 \mathrm{THz}$, respectively.

In conclusion, we have studied the quantitative relation between plasmon resonance versus graphene resonator length and Fermi level, which indicate the existence of both 2D electron systems and massless Dirac electrons. By having two tunable plasmon resonances based on graphene cut-wires 


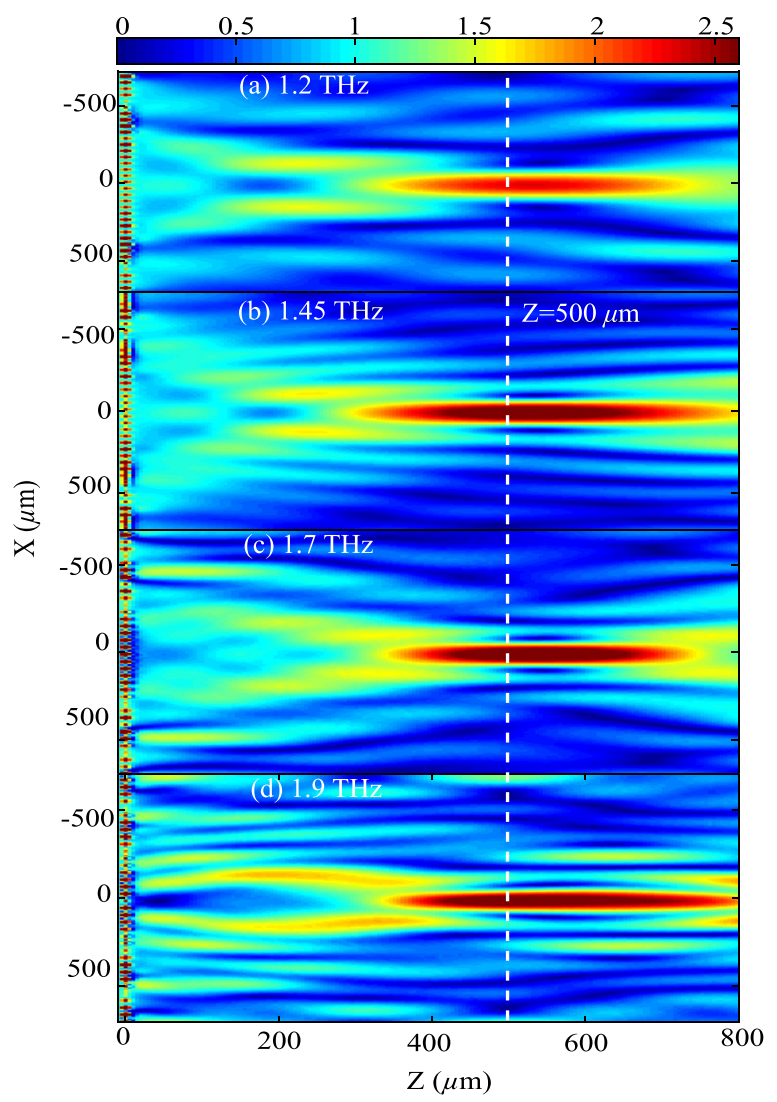

FIG. 4. Electric field amplitude of the metasurface having desired phase profile for (a) $1.2 \mathrm{THz}$, (b) $1.45 \mathrm{THz}$, (c) $1.7 \mathrm{THz}$, and (d) $1.9 \mathrm{THz}$ with the field of the incident waves subtracted.
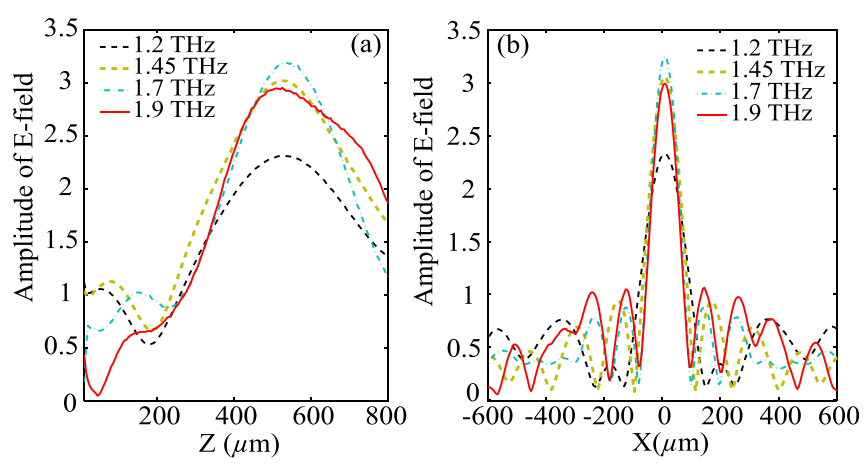

FIG. 5. Electric field amplitude in (a) $Z$ direction (for $X=0 \mu \mathrm{m}$ ) and (b) $X$ direction (when $\mathrm{Z}$ corresponding to the focal points) for $1.2 \mathrm{THz}, 1.45 \mathrm{THz}$, $1.7 \mathrm{THz}$, and $1.9 \mathrm{THz}$, respectively.

in one unit cell, $2 \pi$ phase modulation with minimum reflection efficiency of $48 \%$ can be achieved in a wide range of frequencies. Based on this building block, a metasurface focusing mirror is demonstrated to focus $\mathrm{THz}$ waves from 1.2 to $1.9 \mathrm{THz}$ to the same focal length of $500 \mu \mathrm{m}$. Fabrication of the graphene cut-wires is compatible with current lithography technology, ${ }^{13}$ and using ITO NWhs electrode for gating graphene does not affect $\mathrm{THz}$ performance of the device. These cut-wire resonators could be directly used in 2D metasurfaces, which give us total control of the complete 2D metasurfaces platform for various applications.

This work was partially supported by the Asian Office of Aerospace Research and Development-U.S. Air Force (Grant No. FA2386-15-1-4064).

${ }^{1}$ M. Decker, I. Staude, M. Falkner, J. Dominguez, D. N. Neshev, I. Brener, T. Pertsch, and Y. S. Kivshar, Adv. Opt. Mater. 3, 813 (2015).

${ }^{2}$ N. Yu, P. Genevet, M. Kats, F. Aieta, J.-P. Tetienne, F. Capasso, and Z. Gaburro, Science 334, 333 (2011).

${ }^{3}$ Q. Yang, J. Gu, D. Wang, X. Zhang, Z. Tian, C. Ouyang, R. Singh, J. Han, and W. Zhang, Opt. Express 22, 25931 (2014).

${ }^{4}$ L. Huang, X. Chen, H. Mühlenbernd, H. Zhang, S. Chen, B. Bai, Q. Tan, G. Jin, K.-W. Cheah, C.-W. Qiu, J. Li, T. Zentgraf, and S. Zhang, Nat. Commun. 4, 2808 (2013).

${ }^{5}$ X. Ni, A. Kildishev, and V. Shalaev, Nat. Commun. 4, 2807 (2013).

${ }^{6}$ H.-T. Chen, J. F. O'Hara, A. K. Azad, A. J. Taylor, R. D. Averitt, D. B. Shrekenhamer, and W. J. Padilla, Nat. Photonics 2, 295 (2008).

${ }^{7}$ L. Liu, I. V. Shadrivov, D. A. Powell, R. Raihan, H. T. Hattori, M. Decker, E. Mironov, and D. N. Neshev, IEEE Trans. Tetrahertz Sci. Technol. 3, 827 (2013).

${ }^{8}$ E. Ekmekci, A. Strikwerda, K. Fan, G. Keiser, X. Zhang, G. TurhanSayan, and R. D. Averitt, Phys. Rev. B 83, 193103 (2011).

${ }^{9}$ L. Liu, W.-C. Chen, D. Powell, W. Padilla, F. Karouta, H. Hattori, D. Neshev, and I. Shadrivov, Appl. Phys. Lett. 105, 151102 (2014).

${ }^{10}$ J. Dawlaty, S. Shivaraman, J. Strait, P. George, M. Chandrashekhar, F. Rana, M. Spencer, D. Veksler, and Y. Chen, Appl. Phys. Lett. 93, 131905 (2008).

${ }^{11}$ G. Hanson, J. Appl. Phys. 103, 064302 (2008).

${ }^{12}$ S. Lee, M. Choi, T.-T. Kim, S. Lee, M. Liu, X. Yin, H. Choi, S. Lee, C.-G. Choi, S.-Y. Choi, X. Zhang, and B. Min, Nat. Mater. 11, 936 (2012).

${ }^{13}$ L. Ju, B. Geng, J. Horng, C. Girit, M. Martin, Z. Hao, H. Bechtel, X. Liang, A. Zettl, Y. Shen, and F. Wang, Nat. Nanotechnol. 6, 630 (2011).

${ }^{14}$ Y. Yao, M. Kats, P. Genevet, N. Yu, Y. Song, J. Kong, and F. Capasso, Nano Lett. 13, 1257 (2013).

${ }^{15}$ W. Zhu, F. Xiao, M. Kang, D. Sikdar, and M. Premaratne, Appl. Phys. Lett. 104, 051902 (2014).

${ }^{16}$ Z. Li, K. Yao, F. Xia, S. Shen, J. Tian, and Y. Liu, Sci. Rep. 5, 12423 (2015).

${ }^{17}$ T. Yatooshi, A. Ishikawa, and K. Tsuruta, Appl. Phys. Lett. 107, 053105 (2015).

${ }^{18}$ I. V. Iorsh, I. S. Mukhin, I. V. Shadrivov, P. A. Belov, and Y. S. Kivshar, Phys. Rev. B 87, 075416 (2013).

${ }^{19}$ A. Fallahi and J. Perruisseau-Carrier, Phys. Rev. B 86, 195408 (2012).

${ }^{20}$ Y. Fan, N.-H. Shen, T. Koschny, and C. Soukoulis, ACS Photonics 2, 151 (2015).

${ }^{21}$ C.-S. Yang, T.-T. Tang, P.-H. Chen, R.-P. Pan, P. Yu, and C.-L. Pan, Opt. Lett. 39, 2511 (2014).

${ }^{22}$ C.-S. Yang, M.-H. Lin, C.-H. Chang, P. Yu, J.-M. Shieh, C.-H. Shen, O. Wada, and C.-L. Pan, IEEE J. Quantum Electron. 49, 677 (2013).

${ }^{23}$ F. Karouta, J. Phys. D: Appl. Phys. 47, 233501 (2014).

${ }^{24}$ O. Paul, C. Imhof, B. Reinhard, R. Zengerle, and R. Beigang, Opt. Express 16, 6736 (2008).

${ }^{25}$ H.-S. Chu and C. How Gan, Appl. Phys. Lett. 102, 231107 (2013).

${ }^{26}$ A. Pors, O. Albrektsen, I. Radko, and S. Bozhevolnyi, Sci. Rep. 3, 2155 (2013).

${ }^{27}$ E. H. Hwang and S. Das Sarma, Phys. Rev. B 75, 205418 (2007).

${ }^{28}$ A. Pors, M. Nielsen, R. Eriksen, and S. Bozhevolnyi, Nano Lett. 13, 829 (2013). 